\title{
Robotic-Assisted Surgery Improves the Quality of Total Mesorectal Excision for Rectal Cancer Compared to Laparoscopy: Results of a Case-Controlled Analysis
}

\author{
Pierre Allemann $^{1}$ - Céline Duvoisin ${ }^{1} \cdot$ Luca Di Mare $^{1} \cdot$ Martin Hübner $^{1}$. \\ Nicolas Demartines ${ }^{1} \cdot$ Dieter Hahnloser $^{1}$
}

Published online: 9 November 2015

(C) Société Internationale de Chirurgie 2015

\begin{abstract}
Background The use of a robotic surgical system is claimed to allow precise traction and counter-traction, especially in a narrow pelvis. Whether this translates to improvement of the quality of the resected specimen is not yet clear. The aim of the study was to compare the quality of the TME and the short-term oncological outcome between robotic and laparoscopic rectal cancer resections.

Methods 20 consecutive robotic TME performed in a single institution for rectal cancer (Rob group) were matched 1:2 to 40 laparoscopic resections (Lap group) for gender, body mass index (BMI), and distance from anal verge on rigid proctoscopy. The quality of TME was assessed by 2 blinded and independent pathologists and reported according to international standardized guidelines.

Results Both samples were well matched for gender, BMI (median 25.9 vs. $24.2 \mathrm{~kg} / \mathrm{m}^{2}, p=0.24$ ), and level of the tumor ( 4.1 vs. $4.8 \mathrm{~cm}, p=0.20$ ). The quality of the TME was better in the Robotic group (complete TME: 95 vs. $55 \% ; p=0.0003$, nearly complete TME 5 vs. $37 \% ; p=0.04$, incomplete TME 0 vs. $8 \%, p=0.09$ ). A trend for lower positive circumferential margin was observed in the Robotic group (10 vs. $25 \%, p=0.1)$.

Conclusions These results suggest that robotic-assisted surgery improves the quality of TME for rectal cancer. Whether this translates to better oncological outcome needs to be further investigated.
\end{abstract}

\section{Introduction}

Laparoscopic surgery for rectal cancer remains challenging due to a difficult exposure in a narrow pelvis, due to the need to achieve critical margins and to the requirement of a nerve-sparing dissection. This translates to a high rate of conversion to open surgery [1]. Despite this, laparoscopic surgery is safe and has equivalent short-term oncologic outcomes compared to open surgery [1-3]. Robotic-assisted surgery has been successfully introduced into clinical routine during the past 10 years. This approach claims

Dieter Hahnloser

dieter.hahnloser@chuv.ch

1 Department of Visceral Surgery, University Hospital CHUV, Rue du Bugnon 46, 1011 Lausanne, Switzerland to be superior to open or laparoscopic surgery especially in narrow spaces [4]. This superiority may be due to a better retraction, a perfectly stable vision and the versatility of the articulated instruments (the so-called Endowrist ${ }^{\mathrm{TM}}$ ). However, validation of robotic resection is still poor, particularly in terms of oncological outcomes [5] and contradicting results have been reported in the recent literature, with discrepancies between the quality of the resection and the real clinical benefit, in terms of survival and recurrence rate. The ROLARR trial comparing robotic assisted to laparoscopic rectal cancer resection (NCT01736072) [6] will hopefully answer some of these interrogations.

The short-term oncologic results of the COLOR II trial, comparing laparoscopic to open resection, demonstrated for cancers in the lower third significant less positive circumferential resection margins (CRM) in the laparoscopic 
group ( 9 vs. $21 \%, p=0.013$ ) [2]. A better visualization was claimed to explain this difference. If so, robotic surgery could even be more precise than standard laparoscopy, due to the aforementioned advantages in terms of both visualization and rectum handling in narrow spaces.

The aims of this study were, therefore, to compare the quality of the total mesorectal excision (TME) and the short-term oncological outcomes between robotic and laparoscopic rectal cancer resections.

\section{Patients and methods}

Laparoscopic TME is currently the surgical standard approach for rectal cancer in our institution (Lausanne university hospital). Since 2009, pertinent demographics, peri- and post-operative variables, and long-term follow-up data of all patients undergoing rectal resections were prospectively collected. Since May 2012, a multidisciplinary program of robotic surgery was started and included also robotic TME for rectal cancer. All patients had a standardized preoperative work-up, including digital examination, rigid rectoscopy, complete colonoscopy, thoracic-abdominal CT, and pelvic MRI. The results were then presented in a multidisciplinary tumor board.

There was neither patient selection, nor randomization. Both approaches, including operative technique, set-up details and potential complications were exposed to all patients referred to our department with rectal cancer. After information and open discussion, patients finally decided freely which approach was used, according to its own preference. Written informed consent was obtained before every intervention. The study was approved by the local ethical committee.

\section{Operative technique}

The Da Vinci HD Si system (Intuitive Surgical inc., Sunnyvale, USA) was used. All robotic cases were operated with a dual console system. After sterile draping, the patient was placed in Trendelenburg position. A $12 \mathrm{~mm}$ port was placed open, in the umbilicus. Then, three robotic $8.5-\mathrm{mm}$ cannulas and a $12-\mathrm{mm}$ trocar were placed in a halfcircle fashion, joining the two iliac spines with the umbilicus. An extra 5-mm port was placed in the right upper quadrant for retraction. The robot was then docked between the legs of the patients. The robot was used for inferior mesenteric vessels dissection and TME, using monopolar scissors and bipolar Maryland dissector. The splenic flexure was mobilized laparoscopically using standard instruments.

In the laparoscopic group, a four trocar technique placed in a rhomboid fashion was used. Dissection and vessels ligation was done using a 5-mm monopolar tip Ligasure Advanced (Covidien, Mansfield, USA). The vision was provided by a HD Storz optical system (Karl Storz, Tuttlingen, Germany) using a 30 degree camera.

\section{Study design}

This study was a retrospective analysis of a prospectively maintained database, including all patients operated with the robotic system (Robot group) in a single institution. This population was matched and compared to laparoscopic rectal resection performed during the same time period. One experienced colorectal surgeon $(\mathrm{DH}$, more than 1000 rectal resection) and one experienced robotic visceral surgeon (PA, more than 50 robotic interventions) performed themselves or were present in the operative field of all laparoscopic or robotic operations (teaching university hospital). After blinding for identity and outcomes data, patients were matched manually, with a ratio of $1: 2$, to patients operated laparoscopically (Lap group). The matching criteria were gender, BMI ( $<20$ vs. $20-30$ vs. $>30 \mathrm{~kg} / \mathrm{m}^{2}$ ) and distance of the distal margin of the tumor from the anal verge assessed with rigid proctoscopy $(<5 \mathrm{vs}$. $>5 \mathrm{~cm}$ ). Stage and preoperative treatment were not considered as matching criteria, in order to simplify the process. According to the small sample size, formal propensity score was omitted and replaced by single criteria comparison, using appropriate test (McNemar, Wilcoxon).

The primary endpoint was the quality of the TME. Looking for the highest level of quality, it was defined as the rate of complete TME only (as described by Quirke et al. [7]). The operative specimen was prepared with colored ink by the pathologist on charge. The latter was blinded regarding the surgical approach. Surgeons were not present during the whole pathological process. It was then analyzed macro- and micro-scopically by two independent pathologists and described in a standardized pathological report. In case of discrepancy, a consensus was obtained after free discussion. Secondary endpoints included: the overall survival, the disease-free survival, the rate of positive CRM (defined as $<1 \mathrm{~mm}$ ), the absolute value of CRM, the lymph node harvest count, the 30-day complication rate (surgical and medical), the mortality rate, the operation time, and the conversion rate to open or laparoscopic surgery.

\section{Statistics}

Data were analyzed using STATA version 13.0 (StataCorp, College Station, TX, USA). Categorical data were expressed as percentages and were analyzed using $\mathrm{McNe}$ mar test. Continuous data were expressed as median and range. They were analyzed using Wilcoxon test. Survival 
data were calculated using Kaplan-Meier method and compared using log-rank test. Converted cases were analyzed in an intention-to-treat manner. $P$ values $<0.05$ were considered being statistically significant.

\section{Results}

From May 2012 to January 2014, 46 patients were operated laparoscopically for rectal cancer and 7 had open resection. During the same period, 20 patients were operated using the robotic system. The latter were matched 1:2 to 40 laparoscopic patients. Both groups were well matched (Table 1).

The quality of the TME was significantly better in the Robot group (complete TME: 95 vs. $55 \% ; p=0.0003$ ), as presented in Table 2. A trend for lower positive circumferential margin was observed in the Robot group (10 vs. $25 \%, p=0.1)$. The median number of lymph nodes clearing was similar in both groups.

Concerning perioperative outcomes (Tables 3, 4), both operative time ( 4 h51 vs. $5 \mathrm{~h} 13, p=0.24$ ) and 30 -day complication rate (40 vs. $35 \%, p=0.34$ ) were similar. There was no mortality in both groups. The conversion rate to open surgery seems somewhat lower in the roboticassisted group (5 vs. $20 \%$ ), yet without reaching statistical significance $(p=0.11)$. Reasons for conversion in the Rob group were obesity with inability to achieve inferior mesenteric vessels exposure safely (one case, TME complete) and in the Lap group: obesity with inability to achieve inferior mesenteric vessels exposure safely (two cases, two complete TME), obesity with inability to achieve TME safely (three cases, one TME complete, one TME nearly complete, one incomplete TME), inability to achieve distal clearance safely (two cases, one complete TME, one incomplete TME), bleeding during splenic flexure mobilization (one case, complete TME).

The mean follow-up was similar between the two groups (22.5 \pm 7.0 months, range $9-35$ vs. 31.8 months \pm 17.1 , range $5-66, p=0.09)$. In the robotic group, one local recurrence occurred, 3 months after the intervention. Four patients presented distant recurrence (two in the liver and two in the lungs) and were reoperated. All but one patient (the one with the local recurrence) were alive free of

Table 1 Patients demographics

\begin{tabular}{llll}
\hline & Rob $(n=20)$ & Lap $(n=40)$ & $p$ value \\
\hline Male $(\%)$ & $60 \%$ & $60 \%$ & 0.5 \\
BMI $\left(\mathrm{kg} / \mathrm{m}^{2}\right.$; mean $\left.\pm \mathrm{SD}\right)$ & $25.9 \pm 9$ & $24.2 \pm 7$ & 0.24 \\
Distance tumor from AV $(\mathrm{cm} ;$ mean $\pm \mathrm{SD})$ & $4.1 \pm 1.7$ & $4.8 \pm 2.6$ & 0.20 \\
Age (years, mean $\pm \mathrm{SD})$ & $64 \pm 12$ & & \\
ASA score $(\%)$ & & $8 \%$ & 0.42 \\
1 & $25 \%$ & $67 \%$ & 0.1 \\
2 & $45 \%$ & $25 \%$ & 0.2 \\
3 & $30 \%$ & $0 \%$ & 0.44 \\
4 & $0 \%$ & $24 / 40(60 \%)$ & 0.5 \\
Neoadjuvant radio-chimiotherapy $(\%)$ & $13 / 20(65 \%)$ & $19 / 24(79 \%)$ \\
Long course radiotherapy & $8 / 13(61 \%)$ & 0.37 \\
\hline
\end{tabular}

$B M I$ body mass index $\left(\mathrm{kg} / \mathrm{m}^{2}\right), S D$ standard deviation, $A V$ anal verge

Table 2 Intraoperative data

\begin{tabular}{llll}
\hline & Rob $(n=20)$ & Lap $(n=40)$ & $p$ value \\
\hline OP time (min, mean) & 291 & 313 & 0.24 \\
Conversion rate (\%) & 5 & 20 & 0.11 \\
Blood losses (ml, mean \pm SD) & $58 \pm 76$ & $219 \pm 421$ & 0.39 \\
Resection type & & & $25 / 40$ \\
Low anterior resection & $15 / 20$ & $9 / 40$ & $6 / 40$ \\
Intersphincteric resection & $3 / 20$ & $94 \%$ & 0.25 \\
Abdominoperineal resection & $2 / 20$ & $84 \%$ &
\end{tabular}

$B M I$ body mass index $\left(\mathrm{kg} / \mathrm{m}^{2}\right), S D$ standard deviation, $A V$ anal verge 
Table 3 Tumor characteristics at pathology

\begin{tabular}{|c|c|c|c|}
\hline & Robotic $(n=20)$ & Laparoscopy $(n=40)$ & $p$ value \\
\hline \multicolumn{4}{|l|}{ Quality of the TME } \\
\hline Complete & $95 \%$ & $55 \%$ & $0.0003 *$ \\
\hline Nearly complete & $5 \%$ & $37 \%$ & $0.04 *$ \\
\hline Incomplete & $0 \%$ & $8 \%$ & 0.09 \\
\hline $\mathrm{CRM}(\mathrm{mm}$, mean $\pm \mathrm{SD})$ & $6.4 \pm 3.6$ & $11.2 \pm 10.4$ & 0.38 \\
\hline Positive CRM $(<1 \mathrm{~mm}, \%)$ & $10 \%$ & $25 \%$ & 0.1 \\
\hline Lymph node (nbr, mean \pm SD) & $24 \pm 14$ & $20 \pm 7$ & 0.1 \\
\hline \multicolumn{4}{|l|}{ TRG score (Mandard) } \\
\hline $1 / 5$ & $1 / 13$ & $1 / 24$ & \\
\hline $2 / 5$ & $4 / 13$ & $9 / 24$ & \\
\hline $3 / 5$ & $4 / 13$ & $11 / 24$ & \\
\hline $4 / 5$ & $3 / 13$ & $3 / 24$ & \\
\hline $5 / 5$ & $1 / 13$ & $0 / 24$ & \\
\hline \multicolumn{4}{|l|}{ Tumor stage (UICC) } \\
\hline I & $5 / 20$ & $8 / 40$ & \\
\hline II & $5 / 20$ & $13 / 40$ & \\
\hline III & $7 / 20$ & $15 / 40$ & \\
\hline IV & $3 / 20$ & $4 / 40$ & \\
\hline
\end{tabular}

* Statistically significant results

$T M E$ total mesorectal excision, $C R M$ circumferential resection margin, $S D$ standard deviation, TRG tumor regression score

Table 4 Clinical outcomes

\begin{tabular}{|c|c|c|c|}
\hline & Robotic $(n=20)$ & Laparoscopy $(n=40)$ & $p$ value \\
\hline 30-day morbidity $(\%)$ & $40 \%$ & $35 \%$ & 0.74 \\
\hline \multicolumn{4}{|l|}{ Dindo-Clavien I-II: } \\
\hline Surgical site hematoma & 1 & - & \\
\hline Urinary retention & 1 & 2 & \\
\hline Prolonged ileus & 1 & - & \\
\hline Parastomal hernia & 1 & - & \\
\hline SSI (bedside treatment) & - & 2 & \\
\hline Pulmonary embolism & - & 1 & \\
\hline \multicolumn{4}{|l|}{ Dindo-Clavien III-IV $(n)$} \\
\hline Small bowel obstruction & 2 & 2 & \\
\hline Ileostomy stenosis & 2 & 2 & \\
\hline Anastomotic leak & - & 2 & \\
\hline SSI (reoperation) & - & 3 & \\
\hline Reoperation rate & $15 \%$ & $13 \%$ & 0.38 \\
\hline Readmission rate & $5 \%$ & $12 \%$ & 0.28 \\
\hline Follow-up (months, mean \pm SD [range]) & $22.5 \pm 7.0[9-35]$ & $31.8 \pm 17.1[5-66]$ & 0.09 \\
\hline 2-year overall survival (months) & $100 \%$ & $98 \%$ & 0.67 \\
\hline 2-year disease-free survival (months) & $78 \%$ & $74 \%$ & 0.11 \\
\hline
\end{tabular}

SSI surgical site infection, $S D$ standard deviation

disease, at the last follow-up. In the laparoscopic group, three local recurrences were diagnosed at 10, 18, and 24 months. Only one patient was suitable for re-operation.
Ten patients presented distance recurrence (four livers, four lungs, one liver + lung and one bone metastases) and three of them were re-operated. 30 patients were alive free of 
disease at the last follow-up, 9 patients were alive, with the disease and one patient died from the disease. Both actuarial two-year overall survival (100 vs. $98 \%, p=0.67$ ) and two-year disease-free survival (78 vs. $74 \%, p=0.11$ ) were similar between the two groups.

\section{Discussion}

This case-matched analysis demonstrated that robotic-assisted surgery achieved significantly more complete TME. In other words, the quality of the dissected specimen was better when using the robotic system compared to standard laparoscopic resections, at our institution.

First of all, it should be noted that the rate of complete TME in the laparoscopic group was rather low. When analyzing these disappointing results, we found, however, no difference between the two groups concerning the rate of incomplete TME, which is in fine more relevant for the risk of local recurrence. This discrepancy was explained by a higher rate of nearly complete TME in the laparoscopic group. It is true that there is little difference between complete and nearly complete TME as described by Quirke et al. [7] and this could represent a well-founded criticism of our work. However, as our hypothesis was focusing on the perfection of dissection, only complete TME were considered as a valid target and included in our analysis as the primary outcome. Moreover, less robotic operations were performed by surgeons in training for colorectal surgery specialization and this could also have played some role. However, the senior trainer was always present during the entire operation (robotic and laparoscopic) and took over or converted if the quality of the TME seemed to be compromised due to teaching. We therefore do not believe that teaching should have influenced negatively the quality of the resected specimen, but we cannot formally prove it. However, as a university teaching hospital, training surgeons is very important and the robotic approach will probably increase teaching possibilities in the future, due to the dual console system. In this particular setting, the surgeon and the trainee have exactly the same exposure, vision and instrumental setting, which is not the case during laparoscopic or open operations. Finally, it should be emphasized, that this $55 \%$ rate of complete TME in the laparoscopic group represent an important limitation, indicating that robotic was perhaps not better, but that results of laparoscopic TME should be improved.

Lower conversion rate to open surgery have widely been reported as a major (if not the sole) clear advantage of robotic surgery of rectal cancer [5]. In our own results, this advantage was also observed, but did not reached significant threshold. As the primary endpoint of our study was not the conversion rate, but the quality of the TME, this negative result was probably due to a sample size effect. Interestingly, the rate of incomplete TME seems not being influenced by conversion to open (robotic: 1 conversion, complete TME. Lap group: 8 conversions, 2 incomplete TME, 1 nearly complete TME and 5 complete TME); however, the number of event was hopefully low, precluding formal statistical analysis.

Our findings are concordant with those of Baïk et al. who reported similar rates of complete TME of $93 \%$ [8]. More recently, Luca et al. also reported a rate of complete TME as high as $88 \%$ with robotic-assisted surgery [9]; however, this was a non-comparative study. Such positive results were, however, not described in all series reporting robotic rectal resections. In their report of 20 cases, Barnajian et al. failed to demonstrate any superiority of the robotic approach in terms of TME quality [10].

The reasons are probably variable. By critically reviewing the videos of our procedure, we found that the way the plan of TME was obtained was very different between the two groups. During robotic interventions, the exposition during the dissection was always obtained by pushing the rectal specimen against the opposite direction of the dissection track. Direct traction or grasping of the rectal specimen (observed in some of laparoscopic interventions) was never used, avoiding tearing of the mesorectal fascia. This was particularly the case at the end of the TME plane, approaching the pelvic floor or in obese male patients. Moreover, the stable 3D vision was also found to be of clear advantage, with less camera displacement and less lens blurring.

No statistically significant differences were found in our collective in terms of positive CRM. Due to a retrospective design, a power analysis was not done and this absence of difference could also be due to a small sample size. Actually, a lower rate of positive CRM was described by D'annibale et al. (0 vs. $12 \%, p=0.022$ ) in favor if robotic resections [11]. This was confirmed by a recent metaanalysis published in 2015 [12], including more than 550 robotic cases and reporting statistically significant lower rate of positive CRM using the robotic approach (2.74 vs. $5.78 \%$, OR 0.44, $95 \%$ CI [0.20, 0.96], $p=0.04$ ).

According to Jimenez-Rodrigez [13] and D'annibale [11], the learning curve for robotic TME range from 20 to 25 cases. Our current results are particularly encouraging, as they included our first 20 cases of robotic rectal resections and we already have better specimens compared to laparoscopic surgery. This means that some improvement still can be expected with our next interventions.

The study has the limitation of not being randomized. We, however, attempt to limit this bias by case-matching all robotic resections 1:2 with multiples criteria influencing the dissection especially in the narrow male pelvis. Oncological criteria, such as stage and neoadjuvant 
treatment were not considered as matching criteria, but where well balanced between the two groups. Selection bias was also limited by proposing both techniques (robotic and laparoscopic) for all patients, without any selection criteria. Moreover, all the data were prospectively collected, irrespective of the surgical technique.

This study was performed using the Da Vinci Si system. It was the latest model at the time we started our program. It has the limitation to work in a half-circle working space and need re-docking to obtain comfortable access to the whole abdominal cavity. For this reason, a hybrid robotic and laparoscopic technique was chosen. Because robotic assistance is thought to be advantageous in narrow spaces such as the pelvis and during mesenteric vessels dissection. It is unlikely that robotic-assisted surgery would be superior to laparoscopic surgery for the splenic flexure mobilization, were precision and exposure are less important. This limitation is now surmounted by the new robotic platform.

Knowing that TME completeness and CRM status are both predictors of local recurrence [14], the results presented in the current study and in other reports $[8,11,12]$ opens interesting perspectives in terms of oncological outcomes. Whether better surgical specimens will translated also to better oncological outcomes is actually not clear. In our series, no differences have been found in terms of overall survival and disease-free survival after a mean follow-up of 22.5 and 31.8 months. These results are concordant with the current literature. Indeed, many recent reports-including retrospective or non-comparative prospective trials-have demonstrated to provide equivalent results in terms of overall survival and disease-free survival [15-17]. However, none of them succeed to demonstrate any real advantages for a robotic approach, compared to laparoscopy. Even the most experienced team from Korea [18] reported no difference in terms of 5-year overall survival (92.8\% in the robotic group vs. $93.5 \%$ in the laparoscopic group, $p=0.829$ ) and 5-year disease-free survival (81.9 vs. $78.7 \%, p=0.547$ ), when analyzing an impressive cohort of 133 robotic TME. Finally, this absence of superiority was also highlighted in the metaanalysis of Xiong et al. Even after combining the results of eight recent studies [12], no difference in disease-free survival was detected. In our opinion, it is likely that only large multicentric trial will have enough patients to answer this question. In this perspective, the randomized controlled trial ROLARR will eventually provide some elements of response [6].

\section{Conclusion}

The results of this case-controlled study suggest that robotic-assisted surgery improves the specimen quality of TME for rectal cancer surgery. Whether this translates to better oncological outcome needs to be further investigated on a larger series.

\section{Compliance with ethical standards}

Financial disclosure and conflicts All authors declare to have no biomedical financial interests or potential conflicts of interest of any nature. The study was fully supported by our institution, without any external funding.

\section{References}

1. Guillou PJ, Quirke P, Thorpe H, Walker J, Jayne DG, Smith AM, Heath RM, Brown JM, MRC CLASICC trial group (2005) Shortterm endpoints of conventional versus laparoscopic-assisted surgery in patients with colorectal cancer (MRC CLASICC trial): multicenter, randomised controlled trial. Lancet 365(9472): $1718-1726$

2. van der Pas MH, Haglind E, Cuesta MA, Fürst A, Lacy AM, Hop WC, Bonjer HJ, COlorectal cancer Laparoscopic or Open Resection II (COLOR II) Study Group (2013) Laparoscopic versus open surgery for rectal cancer (COLOR II): short-term outcomes of a randomised, phase 3 trial. Lancet Oncol 14(3): 210-218

3. Kang SB, Park JW, Jeong SY, Nam BH, Choi HS, Kim DW, Lim SB, Lee TG, Kim DY, Kim JS, Chang HJ, Lee HS, Kim SY, Jung KH, Hong YS, Kim JH, Sohn DK, Kim DH, Oh JH (2010) Open versus laparoscopic surgery for mid or low rectal cancer after neoadjuvant chemoradiotherapy (COREAN trial): short-term outcomes of an open-label randomised controlled trial. Lancet Oncol 11(7):637-645

4. Scarpinata R, Aly EH (2013) Does robotic rectal cancer surgery offer improved early postoperative outcomes? Dis Colon Rectum 56(2):253-262

5. Xiong B, Ma L, Zhang C, Cheng Y (2014) Robotic versus laparoscopic total mesorectal excision for rectal cancer: a metaanalysis. J Surg Res 188(2):404-414

6. Collinson FJ, Jayne DG, Pigazzi A, Tsang C, Barrie JM, Edlin R, Garbett C, Guillou P, Holloway I, Howard H, Marshall H, McCabe C, Pavitt S, Quirke P, Rivers CS, Brown JM (2012) An international, multicentre, prospective, randomised, controlled, unblinded, parallel-group trial of robotic-assisted versus standard laparoscopic surgery for the curative treatment of rectal cancer. Int J Colorectal Dis 27(2):233-241

7. Nagtegaal ID, van de Velde CJ, van der Worp E, Kapiteijn E, Quirke P, van Krieken JH, Cooperative Clinical Investigators of the Dutch Colorectal Cancer Group (2002) Macroscopic evaluation of rectal cancer resection specimen: clinical significance of the pathologist in quality control. J Clin Oncol 20(7):1729-1734

8. Baik SH, Kwon HY, Kim JS, Hur H, Sohn SK, Cho CH, Kim H (2009) Robotic versus laparoscopic low anterior resection of rectal cancer: short-term outcome of a prospective comparative study. Ann Surg Oncol 16(6):1480-1487

9. Luca F, Valvo M, Ghezzi TL, Zuccaro M, Cenciarelli S, Trovato C, Sonzogni A, Biffi R (2013) Impact of robotic surgery on sexual and urinary functions after fully robotic nerve-sparing total mesorectal excision for rectal cancer. Ann Surg 257(4):672-678

10. Barnajian M, Pettet D 3rd, Kazi E, Foppa C, Bergamaschi R (2014) Quality of total mesorectal excision and depth of circumferential resection margin in rectal cancer: a matched comparison of the first 20 robotic cases. Colorectal Dis 16(8):603-609

11. D'Annibale A, Pernazza G, Monsellato I, Pende V, Lucandri G, Mazzocchi P, Alfano G (2013) Total mesorectal excision: a comparison of oncological and functional outcomes between 
robotic and laparoscopic surgery for rectal cancer. Surg Endosc 27(6): 1887-1895

12. Xiong B, Ma L, Huang W, Zhao Q, Cheng Y, Liu J (2015) Robotic versus laparoscopic total mesorectal excision for rectal cancer: a meta-analysis of eight studies. J Gastrointest Surg 19(3):516-526

13. Jiménez-Rodríguez RM, Díaz-Pavón JM, de la Portilla de Juan F, Prendes-Sillero E, Dussort HC, Padillo J (2013) Learning curve for robotic-assisted laparoscopic rectal cancer surgery. Int $\mathbf{J}$ Colorectal Dis 28(6):815-821

14. Trakarnsanga A, Gonen M, Shia J, Goodman KA, Nash GM, Temple LK, Guillem JG, Paty PB, Garcia-Aguilar J, Weiser MR (2013) What is the significance of the circumferential margin in locally advanced rectal cancer after neoadjuvant chemoradiotherapy? Ann Surg Oncol 20(4):1179-1184

15. Baik SH, Kim NK, Lim DR, Hur H, Min BS, Lee KY (2013) Oncologic outcomes and perioperative clinicopathologic results after robot-assisted tumor-specific mesorectal excision for rectal cancer. Ann Surg Oncol 20(8):2625-2632

16. Pigazzi A, Luca F, Patriti A, Valvo M, Ceccarelli G, Casciola L, Biffi R, Garcia-Aguilar J, Baek JH (2010) Multicentric study on robotic tumor-specific mesorectal excision for the treatment of rectal cancer. Ann Surg Oncol 17(6):1614-1620

17. Yoo BE, Cho JS, Shin JW, Lee DW, Kwak JM, Kim J, Kim SH (2015) Robotic versus laparoscopic intersphincteric resection for low rectal cancer: comparison of the operative, oncological, and functional outcomes. Ann Surg Oncol 22(4): 1219-1225

18. Park EJ, Cho MS, Baek SJ, Hur H, Min BS, Baik SH, Lee KY, Kim NK (2015) Long-term oncologic outcomes of robotic low anterior resection for rectal cancer: a comparative study with laparoscopic surgery. Ann Surg 261(1):129-137 